\title{
Transvaginal ultrasonography in ovarian cancer screening: current perspectives
}

This article was published in the following Dove Press journal:

International Journal of Women's Health

20 December 2013

Number of times this article has been viewed

\author{
John $R$ van Nagell Jr \\ John T Hoff \\ Department of Obstetrics and \\ Gynecology, University of Kentucky \\ Chandler Medical Center/Markey \\ Cancer Center, Lexington, KY, USA
}

Correspondence: John R van Nagell Jr Division of Gynecologic Oncology, University of Kentucky Markey Cancer Center, 800 Rose Street, Lexington, KY 40536-0293, USA

Tel +l 8593235553

Fax +18593231018

Email jrvann2@email.uky.edu
Abstract: Transvaginal ultrasonography (TVS) is an integral part of all major ovarian cancer screening trials. TVS is accurate in detecting abnormalities in ovarian volume and morphology, but is less reliable in differentiating benign from malignant ovarian tumors. When used as the only screening test, TVS is sensitive, but has a low positive predictive value. Therefore, serum biomarkers and tumor morphology indexing are used together with TVS to identify ovarian tumors at high risk for malignancy. This allows preoperative triage of high-risk cases to major cancer centers for therapy while decreasing unnecessary surgery for benign disease. Ovarian cancer screening has been associated with a decrease in stage at detection in most trials, thereby allowing treatment to be initiated when the disease is most curable.

Keywords: ovarian cancer, ultrasound, screening, serum Ca-125

\section{Introduction}

Ovarian cancer remains a major health problem worldwide, with over 225,000 new cases and 140,000 deaths reported annually., ${ }^{1,2}$ Symptoms associated with ovarian cancer are often nonspecific, and the majority of patients continue to present with advanced disease, where the cost of treatment is high and the survival rate is low. Although early stage ovarian cancer is highly curable with conventional treatment, it is estimated that only $15 \%$ of patients have their disease confined to the ovary at the time of diagnosis. ${ }^{5}$

Many investigators believe that earlier detection is the most effective means to reduce ovarian cancer mortality. For example, it has been estimated that if $75 \%$ of ovarian cancer cases (rather than the present $25 \%$ ) could be detected at stage I or II disease, the number of deaths from this disease would be reduced by half. ${ }^{6}$

Screening is defined as the application of a test or combination of tests to an asymptomatic at-risk population in an attempt to detect a disease at an earlier and more curable stage. A disease that theoretically should benefit from screening has the following characteristics: 1) it should be prevalent in the population to be screened, 2) it should be a major health problem, 3) it should have a significant preclinical stage during which detection through screening is possible, and 4) it should be significantly more curable when diagnosed at an early stage than at an advanced stage. ${ }^{7}$

Ovarian cancer fulfills many of these characteristics, but the duration of its preclinical phase is variable and often unknown. Ovarian cancer is the fifth-leading cause of cancer mortality in females in the US, and often affects women who are well educated and of upper-socioeconomic status. Although this disease is not prevalent 
in the general population, it is significantly more common in women over 50 years of age (incidence 55 in 100,000), ${ }^{8,9}$ and in those with a documented family history of breast or ovarian cancer. In the US, for example, the lifetime risk of ovarian cancer increases from $1.2 \%$ in the general population to $5.0 \%$ in women who have one first-degree relative with the disease. ${ }^{10}$ The estimated lifetime risk of ovarian cancer may be as high as $46 \%$ in women who are $B R C A 1$-positive, and $20 \%$ in women who are $B R C A 2$-positive. ${ }^{11}$ For this reason, all women over the age of 50 years or those over the age of 25 years with a documented family history of ovarian cancer usually are eligible to participate in screening trials.

The pathogenesis of ovarian cancer and therefore the duration of its preclinical stage is variable. Traditionally, epithelial ovarian cancers were thought to arise through changes in the ovarian surface epithelium-lining inclusion cysts formed at the time of ovulation. ${ }^{11,12}$ Recently, Crum et al reported that certain epithelial ovarian cancers may arise from the epithelium of the distal fallopian tube. Tubal intraepithelial carcinoma was noted in the distal fimbria of $57 \%-100 \%$ of women with BRCA1 or BRCA2 mutations and in $47 \%$ of patients with primary peritoneal cancer. ${ }^{14,15}$ p53 mutation analysis was identical in the tubal intraepithelial carcinoma and remote invasive tumors from the same patient, supporting a genetic link between the two. ${ }^{15}$ Kurman and Shih have proposed that there are two types of epithelial ovarian cancers. ${ }^{16}$ Type I cancers are low-grade serous or endometrioid ovarian malignancies, probably arising from neoplastic transformation of the ovarian surface and epithelium. Type I cancers are genetically similar to epithelial ovarian tumors of low malignant potential, and have a prolonged preclinical phase during which screening intervention should be effective. Type II cancers are highgrade, poorly differentiated, serous tumors, many of which arise from the tubal epithelium. These tumors have a shorter preclinical phase, and may require more frequent screening to achieve early detection.

Finally, early stage ovarian cancer is significantly more curable than late-stage ovarian cancer. For example, the 5-year survival of patients with stage I epithelial ovarian cancer is approximately $95 \%$ at major cancer centers, compared to $30 \%$ for patients with stage III disease. ${ }^{17}$ Furthermore, two-thirds of patients with advanced-stage ovarian cancer who are alive at 5 years have recurrent cancer, so the longterm cure rate for these patients is only $10 \%$.

A screening test also must meet certain standards if it is to be effective. An optimal screening test should be easy to perform, time-efficient, and well accepted by those being screened. Also, it should be inexpensive and associated with few side effects, so that screening compliance is high. Finally, the results of the test should be easy to interpret, with minimal interobserver variation. To be effective, a screening test should be sensitive, specific, and associated with a high positive predictive value (PPV) and a high negative predictive value (NPV). The statistical definitions used in ovarian cancer screening are presented in Table 1. A true-positive screen is defined as histologically confirmed ovarian cancer in a woman with an abnormal test. In contrast, a true-negative screen is the absence of ovarian cancer for at least 12 months after a normal test. A false-negative screen is the occurrence of histologically confirmed invasive ovarian cancer within 12 months after a normal scan, whereas a false-positive screen is the absence of ovarian cancer in a patient with a positive screen. Regular screening should lower stage at detection and increase disease-specific ovarian cancer survival. Finally, ovarian cancer mortality should be lower in the screened population than in a similar population of unscreened women. ${ }^{7}$

Transvaginal sonography (TVS) is an integral part of virtually every ovarian cancer screening algorithm, whether it is used as the initial screening test or as a secondary test in women with an elevated biomarker profile. TVS, performed with a 5-7.5 $\mathrm{mHz}$ vaginal probe, generates accurate ovarian images that can be used to detect early changes in ovarian morphology and volume not appreciated on clinical examination. Ovarian volume is calculated using the prolate ellipsoid formula (length $\times$ width $\times$ height $\times 0.523$ ). Criteria for ovarian abnormality vary according to each screening trial, but usually involve both volume and morphology. Ovarian volumes $>20 \mathrm{~cm}^{3}$ in premenopausal women and $>10 \mathrm{~cm}^{3}$ in postmenopausal women are defined as abnormal, because these values are more than two standard deviations above the published mean ovarian volumes for normal women in these two groups. ${ }^{18}$ Morphologic abnormality is based on the presence of solid areas or papillary projections from the

Table I Statistical definitions used in ovarian cancer screening

\begin{tabular}{lll}
\hline Term & Screen & Findings \\
\hline TP & Positive & Histology confirms ovarian cancer \\
FP & Positive & Benign ovarian histology \\
TN & Negative & $\begin{array}{l}\text { No evidence of disease I2 months after } \\
\text { negative screen }\end{array}$ \\
FN & Negative & $\begin{array}{l}\text { Ovarian cancer diagnosed within I2 months } \\
\text { of negative screen }\end{array}$ \\
\hline
\end{tabular}

Notes: Sensitivity, TP/(TP + FN); specificity TN/(TN + FP); positive predictive value, $\mathrm{TP} /(\mathrm{TP}+\mathrm{FP})$; negative predictive value, $\mathrm{TN} /(\mathrm{TN}+\mathrm{FN})$.

Abbreviations: FN, false negative; FP, false positive; TN, true negative; TP true positive. 
cyst wall in a complex cystic ovarian tumor or a solid ovarian tumor with an abnormally increased volume (Figure 1). There are a number of quantitative indexes relating ovarian tumor morphology to risk of malignancy. ${ }^{19-21}$ The tumormorphology index reported by Ueland et $\mathrm{al}^{21}$ is illustrated in Figure 2. Numeric scores (1-5) are given in the categories of tumor volume and tumor morphology, with a combined total score ranging from 0 to 10 . This index was applied to a study population of 442 ovarian tumors, which included 53 ovarian cancers. ${ }^{21}$ Using a morphology-index value $\geq 5$ as indicative of malignancy was associated with the following statistical parameters: sensitivity $98 \%$, specificity $81 \%$, positive predictive value $41 \%$, and negative predictive value $99 \%$. Recent data suggest that unilocular ovarian cysts or septated ovarian cysts are rarely if ever malignant. ${ }^{22,23}$ Long-term follow-up of more than 3,200 unilocular cystic ovarian tumors $<10 \mathrm{~cm}$ diameter detected by screening revealed that the risk of malignancy in these tumors was essentially nonexistent. ${ }^{22}$ Women with these tumors were followed with periodic ultrasound examinations for an average of 6.3 years, and none developed ovarian cancer. In a subsequent investigation, ${ }^{23} 2,870$ septated cystic ovarian tumors detected by screening were followed every 4-6 months by TVS for an average of 6.4 years. Thirtyeight percent of these tumors resolved spontaneously, and no patient developed ovarian cancer. Therefore, unilocular or septated ovarian cysts $<10 \mathrm{~cm}$ diameter detected by screen- ing are no longer considered abnormal and are not removed surgically.

For screening to be effective, there should be a standard evaluation and treatment algorithm applied to all women with a screen-detected ovarian abnormality. The evaluation algorithm used in the University of Kentucky Screening Trial is illustrated in Figure 2. Women with an ovarian abnormality on ultrasound screening are asked to return for repeat sonography in 4-6 weeks. If the ovarian abnormality is persistent on repeat sonography, a serum Ca-125 is obtained and ovarian tumor indexing is performed. Unilocular or septated cystic ovarian tumors $<10 \mathrm{~cm}$ in women with a normal serum $\mathrm{Ca}-125$ are followed without surgery by ultrasound examinations at 6-month intervals. Women with a persisting solid or complex ovarian mass and an elevated or rising serum $\mathrm{Ca}-125$ level are advised to have laparoscopic tumor removal. Patients with an ovarian malignancy on frozen-section histologic evaluation at the time of laparoscopy undergo immediate laparotomy with tumor cytoreduction and staging. Recently, McDonald et $\mathrm{al}^{24}$ evaluated the combination of tumor morphology generated from sonographic images and serum $\mathrm{Ca}-125$ as a means to predict risk of malignancy in ovarian tumors. After multivariable regression analysis, women with an ovarian tumor having complex or solid morphology and a serum Ca-125 value greater than $35 \mathrm{u} / \mathrm{mL}$ were defined as being at high risk

\begin{tabular}{|c|c|c|c|}
\hline 0 & \begin{tabular}{c} 
Benign \\
simple cyst \\
\hline 1
\end{tabular} & $\begin{array}{c}\text { Benign } \\
\text { hemornagic } \\
\text { cyst }\end{array}$ \\
\hline 3 & $\begin{array}{c}\text { Benign cyst with } \\
\text { septation(s) } \\
\text { Malignancy with } \\
\text { papillary } \\
\text { projections }\end{array}$ \\
\hline 5 & $\begin{array}{c}\text { Malignancy } \\
\text { with solid } \\
\text { components } \\
\text { Solid } \\
\text { malignancy } \\
\text { with ascites }\end{array}$ \\
\hline 5
\end{tabular}

Figure I Sonographic images of benign and malignant ovarian morphology. Numeric representation of increasing morphologic complexity is noted in the first column. 
Tumor volume

\begin{tabular}{|l|c|c|}
\hline 0 & $<10 \mathrm{~cm}^{3}$ & \\
\hline 1 & $10-50 \mathrm{~cm}^{3}$ & \\
\hline 2 & $>50-100 \mathrm{~cm}^{3}$ & \\
\hline 3 & $>100-200 \mathrm{~cm}^{3}$ & \\
\hline 4 & $>500 \mathrm{~cm}^{3}$ & \\
\hline & & \\
\hline
\end{tabular}

Figure 2 The University of Kentucky Ovarian Tumor Morphology Index.

Notes: Copyright (C) 2003, with permission from Elsevier. Reprinted from Ueland FR, DePriest PD, Pavlik EJ, et al. Preoperative differentiation of malignant from benign ovarian tumors: the efficacy of morphologic indexing and Doppler flow sonography. Gynecol Oncol. 2003;91:46-50.21

for ovarian cancer. This definition of high risk was evaluated in 395 patients undergoing surgery for an ovarian tumor, 118 of whom had ovarian cancer. This definition had a PPV of $84.7 \%$, an NPV of $92.4 \%$, and correctly identified $77.3 \%$ of patients with stage I and II ovarian cancer and $98.6 \%$ of patients with stage III and IV ovarian cancer.

\section{Analysis of ovarian cancer screening trials}

There are four large ovarian cancer screening trials reported that have TVS as a major component of the screening algorithm (Table 2). The Prostate, Lung, Colorectal and Ovarian (PLCO) trial in the US was a randomized controlled trial of 78,216 women aged 55-74 years assigned to receive either annual screening with TVS and serum Ca-125 for 4 years or their usual gynecologic care. ${ }^{25,26}$ Ultrasound findings considered abnormal included: 1) an ovarian volume $>10 \mathrm{~cm}^{3}$, 2 ) an ovarian cyst volume $>10 \mathrm{~cm}^{3}, 3$ ) any solid area or papillary projection extending into the cavity of a cystic ovarian tumor of any size, or 4) any mixed (solid and cystic) component within a cystic ovarian tumor. Participants and their physicians received written notification of screening results within 3 weeks of testing. However, the evaluation and treatment of each patient with a screening abnormality was left to the discretion of her local physician. Seventeen ovarian tumors of low malignant potential were detected, but were not 
Table 2 Ovarian cancer screening trials utilizing transvaginal sonography

\begin{tabular}{|c|c|c|c|c|c|c|c|c|c|c|}
\hline $\begin{array}{l}\text { Screening } \\
\text { trial }\end{array}$ & Years & $\begin{array}{l}\text { Control } \\
\text { group }\end{array}$ & $\begin{array}{l}\text { Study } \\
\text { design }\end{array}$ & $\begin{array}{l}\text { Screening } \\
\text { test(s) }\end{array}$ & $\begin{array}{l}\text { Number } \\
\text { screened/ } \\
\text { detected }\end{array}$ & $\begin{array}{l}\text { Invasive } \\
\text { cancers }\end{array}$ & $\begin{array}{l}\text { Stages I } \\
\text { and II }\end{array}$ & $\begin{array}{l}\text { Stages III } \\
\text { and IV }\end{array}$ & $\begin{array}{l}\text { Stage } \\
\text { shift }\end{array}$ & $\begin{array}{l}\text { Survival } \\
\text { benefit }\end{array}$ \\
\hline PLCO (USA) $)^{26}$ & $|993-200|$ & $(+)$ & $\begin{array}{l}\text { Randomized } \\
\text { control }\end{array}$ & $\begin{array}{l}\text { Ultrasound } \\
\mathrm{Ca}-125\end{array}$ & 34,253 & 212 & 47 (22\%) & 163 (77\%) & $(-)$ & $(-)$ \\
\hline \multirow[t]{2}{*}{$\begin{array}{l}\text { UKC-TOCS } \\
(\text { UK })^{27}\end{array}$} & $200 \mathrm{I}-2005$ & $(+)$ & $\begin{array}{l}\text { Randomized } \\
\text { control }\end{array}$ & $\begin{array}{l}\text { Ultrasound } \\
\mathrm{Ca}-125\end{array}$ & 50,078 & 34 & $47 \%$ & $53 \%$ & $(+)$ & $\begin{array}{l}\text { Analysis } \\
\text { pending }\end{array}$ \\
\hline & & & & $\begin{array}{l}\text { Ultrasound } \\
\text { alone }\end{array}$ & 48,230 & 24 & $50 \%$ & $50 \%$ & $(+)$ & $\begin{array}{l}\text { Analysis } \\
\text { pending }\end{array}$ \\
\hline $\begin{array}{l}\text { Multicenter } \\
(\text { Japan })^{28}\end{array}$ & $1985-1999$ & $(+)$ & $\begin{array}{l}\text { Randomized } \\
\text { control }\end{array}$ & $\begin{array}{l}\text { Ultrasound } \\
\text { Ca- } 125\end{array}$ & 41,688 & 27 & $67 \%$ & $33 \%$ & $(+)$ & $\begin{array}{l}\text { Analysis } \\
\text { pending }\end{array}$ \\
\hline $\begin{array}{l}\text { University } \\
\text { of Kentucky } \\
(\text { USA })^{29}\end{array}$ & $1987-2013$ & $(+)$ & $\begin{array}{l}\text { Population } \\
\text { control }\end{array}$ & Ultrasound & $4 I, 4 \mid 3$ & 53 & $68 \%$ & $32 \%$ & $(+)$ & $(+)$ \\
\hline
\end{tabular}

Abbreviations: PLCO, Prostate, Lung, Colorectal and Ovarian; UKC-TOCS, UK Collaborative Trial of Ovarian Cancer Screening.

considered as ovarian malignancies in the analysis presented. Seventy-one percent of women whose ovarian cancers were detected by TVS alone had stage I or II disease, but the PPV of TVS varied from only $0.7 \%$ to $1.6 \%$ for each year of the trial, and the ratio of surgeries to screen-detected ovarian cancers was 19.5 to 1 . There was no evidence of a shift to earlier stage disease associated with screening in this trial, and survival rates were similar in the screening and usual-care arms. Unfortunately, there was no uniform evaluation and treatment algorithm followed in patients with screen-detected cancers in this trial; patients in the screening arm could be treated up to 9 months after detection and remain in the study.

The largest ongoing ovarian screening trial is the UK Collaborative Trial of Ovarian Cancer Screening (UKCTOCS). ${ }^{27}$ In this trial, a total of 202,638 postmenopausal women ages 50-74 years were randomly assigned to 1) no treatment $(\mathrm{n}=101,359), 2)$ annual $\mathrm{Ca}-125$ screening with TVS as a second-line test ( $\mathrm{n}=50,078)$, or 3 ) annual screening with TVS alone $(n=48,230)$. Ovarian volume was measured using the prolate ellipsoid formula as previously mentioned, and ovarian morphology was classified as normal, a simple cyst, or complex ovarian mass including any nonuniform echogenicity. Ascites was noted when there was a maximum vertical fluid measurement $\geq 10 \mathrm{~mm}$. An abnormal scan was defined as the presence of complex morphology in one or both ovaries, a simple cyst $>60 \mathrm{~cm}^{3}$ in volume, or ascites. A woman with an abnormal primary screen had a repeat ultrasound examination in 6-8 weeks, and if the repeat scan was abnormal, she was referred for clinical assessment. Clinical assessment included a serum Ca-125, repeat TVS, Doppler studies, and computed tomography/magnetic resonance imaging scans of the abdomen and pelvis. Of the 48,230 women who underwent ultrasound alone 2,774 (5.7\%) were classified as abnormal and had a repeat scan. There was a persisting ovarian abnormality on the repeat scan in 1,824 women. These women then underwent clinical assessment, and 845 $(1.8 \%)$ had surgery. Forty-five of these women had malignant neoplasms of the ovary, 23 of which were borderline tumors. The ratio of surgeries to screen detected cancers in the ultrasound-alone arm of this trial was 18.8 to 1 . Fifty percent of primary invasive ovarian or tubal malignancies detected by ultrasound screening alone had stage I or II disease versus $26 \%$ in the control cases detected clinically, so screening produced a significant increase in the detection of early stage ovarian malignancy. In the multimodality-screening arm of this trial, ultrasound was performed only in women whose Ca-125 values placed them in an intermediate or high risk for ovarian cancer. Of the 50,078 women in the multimodalityscreening arm, 409 (0.8\%) had TVS, and 97 underwent surgery after clinical assessment. Thirty-four patients had ovarian cancer, 16 of whom (47\%) had stage I or II disease. The ratio of surgeries to screen-detected cancers in this arm of the trial was 2.8 to 1 . The UKC-TOCS trial is ongoing, and the effect of screening on ovarian cancer mortality will be published after data analysis is complete.

The multicenter ovarian cancer screening trial in $\operatorname{Japan}^{28}$ was a prospective randomized trial conducted between 1985 and 1999 in which asymptomatic postmenopausal women were assigned either to a screening $\operatorname{arm}(n=41,688)$ or a control arm $(n=40,799)$. Women in the screening arm received an annual pelvic examination, an annual pelvic ultrasound, and a serum Ca-125. During the ultrasound examination, each ovary was measured in three dimensions, and ovarian morphology was documented. Ultrasound findings were classified 
as follows: 1) normal ovary, largest diameter $<4 \mathrm{~cm}$ with normal morphology, 2) benign impression, ovarian length $\geq 4 \mathrm{~cm}$ with simple morphology, or 3) malignant impression, ovarian length $\geq 4 \mathrm{~cm}$ with complex morphology. Management, including surgical intervention, was at the discretion of a gynecologic oncologist. A total of 103 patients thought to be at high risk for ovarian cancer on the basis of ultrasound findings were evaluated by a gynecologic oncologist, and 64 underwent surgery. Twenty patients were found to have primary ovarian cancer, and ten had metastatic disease to the ovary. As in all the screening trials other than the PLCO trial, regular ultrasound screening was associated with a decrease in stage at detection. In the Japanese trial, $63 \%$ of ovarian cancer patients detected by screening had stage I disease versus $38 \%$ in the control arm. Likewise, optimal tumor debulking was achieved more frequently in women whose ovarian cancer was detected by screening. Assessment of the long-term effect of screening on ovarian cancer mortality is presently in progress.

The University of Kentucky Ovarian Cancer Screening Trial (UKOCS) has been in progress since 1987, and has enrolled 41,413 women. ${ }^{29}$ Eligibility criteria include all women over the age of 50 years and women above 25 years of age with a documented family history of ovarian cancer. This trial does not have a "no screening" control arm into which women are prospectively enrolled. Rather, data concerning women receiving screening is compared to a control population of women from the same geographic area, receiving the same treatment protocols, at the same hospital, over the same time period who did not receive screening. In this screening trial, all women with an abnormality on TVS are evaluated and treated according to a standard protocol (see Figure 2). Women with an abnormal screen have a repeat screen in 4 weeks. If the repeat screen is abnormal, a serum Ca-125 is obtained, Doppler analysis of tumor blood flow is performed, and tumor morphology indexing is completed (see Figure 3). If these studies indicate that an ovarian tumor is at high risk for malignancy, laparoscopic tumor removal is performed as soon as possible. The time between ovarian tumor detection and surgical removal is designed not to exceed 8 weeks. To date, 53 primary epithelial ovarian malignancies have been detected in the UKOCS trial, 68\% of which were limited to the ovary or pelvis (stage I or II disease). Twelve women developed ovarian cancer within

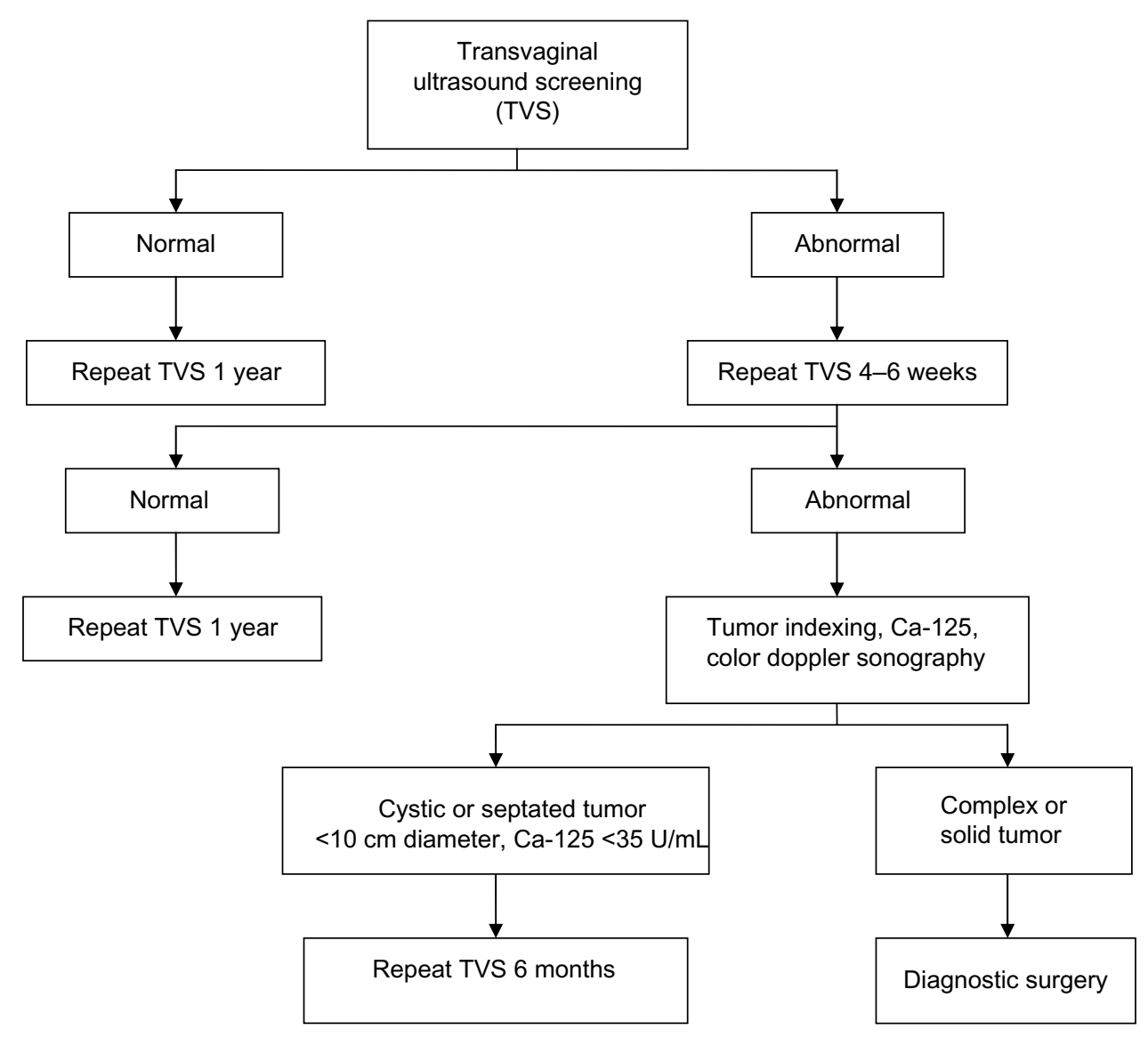

Figure 3 Evaluation algorithm for women enrolled in the University of Kentucky Ovarian Cancer Screening Trial. 
12 months of a normal screen (interval cancers). Women whose ovarian cancers were diagnosed by screening had earlier-stage disease at detection (68\% stage I or II disease) than those who did not receive screening $(27 \%$ stage I or II disease, $P<0.01)$. In addition, there was a substage shift within stage III in that more women in the screening group had stage IIIA disease. The 5-year survival of all women whose epithelial ovarian cancers were detected by screening including interval (false-negative) cancers was $74.8 \% \pm 6.6 \%$ compared to $53.7 \% \pm 2.3 \%$ for women with clinically detected ovarian cancers treated at the same institution during the same time period with identical surgical and chemotherapy protocols $(P<0.01)$.

\section{Cost of screening}

The cost of screening varies according to the individual trial and the specific algorithm employed. The cost of each ultrasound is approximately $\$ 40$ when performed in highvolume screening centers using modern two-dimensional ultrasound equipment. ${ }^{30}$ Single-biomarker testing is less expensive than multiple-marker panels, but even the cost of a single Ca-125 determination varies significantly from one institution to another. With developing technology, every effort must be made to reduce the cost of biomarker testing. This is particularly important, since sequential biomarker and ultrasound testing are being utilized as a means to increase the PPV of screening and reduce unnecessary surgery. The cost of screening must be evaluated in the context of treatment costs for patients with ovarian cancer. Analysis of financial data, including inpatient, outpatient, pharmacy, and physician charges, indicates that the total cost of treatment for a patient with stage IIIC epithelial ovarian cancer is in excess of $\$ 200,000 .{ }^{31}$ Much of this cost is related to the treatment of recurrent ovarian cancer, which is common in patients initially detected with advanced-stage disease. This analysis did not place a financial value on years of lost productivity from disease-specific mortality.

\section{Future ovarian cancer screening trials}

Fundamental questions remain concerning who should be screened, the frequency of screening, and the optimal order of tests within a specific screening algorithm. Although ovarian cancer is a leading cause of cancer mortality, it is a relatively rare disease, occurring with an incidence of only 55 in 100,000 in high-risk age-groups. The incidence of ovarian cancer is higher in postmenopausal women, women with a family history of ovarian cancer, and women with certain genetic mutations, such as $B R C A 1, B R C A 2$, and Lynch II syndrome. As a result, eligibility in most screening trials is limited to postmenopausal women or women with a documented family history of ovarian cancer over the age of 25 years. Further, epidemiologic research is needed to determine additional demographic and molecular genetic factors that predispose women in the general population to be at increased risk of ovarian cancer. The type and frequency of screening can then be designed based on an individual's ovarian cancer-risk profile. As specific at-risk populations are defined more clearly, the cost of screening should decrease.

The origin of certain ovarian malignancies from the fallopian tube epithelium ${ }^{13}$ may make these tumors more difficult to detect at an early stage. Studies are in progress to assess the diagnostic accuracy of advanced ultrasound in detecting early morphologic abnormalities in the fallopian tube, but the results have yet to be published. The exact frequency of ovarian cancers that arise from the fallopian tube epithelium is unknown, but future screening trials may require evaluation of both tubal and ovarian morphology.

TVS has been accurate in identifying minimal changes in ovarian volume and morphology, but has not been reliable in differentiating benign from malignant ovarian tumors. Morphology indexing (MI) has been developed as a method to quantitate changes in tumor morphology and relate them to risk of malignancy (Figure 2). Ovarian tumors without solid areas or papillary projections have been shown to be rarely if ever neoplastic, and can be followed sonographically without surgery. However, the histology of complex ovarian tumors is more difficult to predict from ultrasound images. Recently, serial MI has been used to distinguish benign from malignant ovarian tumors. MI values were found to increase with time in ovarian cancers, but decreased or remained stable in benign ovarian tumors. ${ }^{32}$ Serial MI determinations at biweekly or monthly intervals may be incorporated into future screening trials in women with persisting complex ovarian tumors as a means to increase PPV of screening in these patients.

Another area of recent research interest is contrastenhanced TVS using microbubble contrast-agent particles. ${ }^{33}$ These particles have a dynamic response in the ultrasonic field, and can more accurately define tumor neovascularity. This technology was evaluated in a preliminary study involving 23 morphologically abnormal ovarian tumors, nine of which were malignant. ${ }^{34}$ There was a statistically significant difference in contrast-enhancement kinetics between benign and malignant ovarian tumors. Specifically, peak enhancement, half-washout time, and area under the enhancement 
curve were all higher in malignant ovarian tumors when compared to benign lesions. Using a diagnostic criterion of an area under the curve $>787$ seconds $^{1}$ as indicative of malignancy achieved a sensitivity of $100 \%$ and a specificity of $96.2 \%$. These results are interesting, and await confirmation in prospective clinical trials.

It is worth reemphasizing that a standard evaluation and treatment protocol should be applied uniformly to all patients with a screen-detected ovarian abnormality. Such a protocol should be designed such that operative intervention is recommended only in patients who after repeat testing are at significant risk for ovarian cancer. Also, surgery should be performed in a timely fashion, preferably within 8 weeks of diagnosis, in order to preserve the benefits of early detection. Two prerequisites for a successful screening test are that it lower stage at detection and reduce disease-specific mortality. ${ }^{7}$ TVS has been shown to lower stage at detection in most trials. However, it is difficult to prove that screening decreases ovarian cancer mortality unless an effective evaluation and treatment algorithm is applied uniformly to women in whom screening has identified an ovarian abnormality.

Finally, the optimal combination of TVS with other screening tests is undergoing continued evaluation. Sonography alone is unreliable in detecting primary peritoneal cancer or ovarian cancer in which there is no volume or morphologic abnormality of the ovary. Therefore, some algorithms, such as the multimodal screening arm of the UKC-TOCS trial, utilize TVS as a secondary test in women designated as high risk on the basis of their biomarker profile. ${ }^{27}$ This approach increases the PPV of TVS. For example, 42 of the 97 patients designated as high risk by biomarker profile in the UKC-TOCS trial had ovarian cancer at the time of surgery $(\mathrm{PPV}=43.2 \%)$. In contrast, only 45 of 845 patients had ovarian cancer $(\mathrm{PPV}=2.3 \%)$ when ultrasound was used as the sole screening modality. The sensitivity of biomarkers in detecting early stage ovarian cancer is low, however, and these cancers would be missed if they did not produce detectable marker levels in the serum. Hirai et al, for example, reported that only $40 \%$ of stage IA ovarian cancers have an elevated serum Ca-125 $5^{35}$. As has been mentioned, when TVS is used as the primary screening test, repeat sonography and biomarker analysis should be performed prior to recommending surgery, so that operative intervention can be avoided in women whose ovarian abnormalities are resolving spontaneously.

Although TVS has known limitations as a screening method for ovarian cancer, it remains an integral part of all screening trials. The majority of these trials have reported that annual screening lowers stage of detection, and therefore should reduce ovarian cancer mortality. Research is in progress to develop new diagnostic tests, which when combined with ultrasound will increase the accuracy and PPV of screening. Early detection remains of fundamental importance, since it allows effective treatment to be initiated when ovarian cancer is most curable.

\section{Disclosure}

The authors report no conflicts of interest in this work.

\section{References}

1. Siegel R, Naishadham D, Jemal A. Cancer statistics, 2013. CA Cancer J Clin. 2013;63:11-30.

2. Jemal A, Bray F, Center MM, Ferlay J, Ward E, Forman D. Global cancer statistics. CA Cancer J Clin. 2011;61:69-90.

3. Goff BA, Mandel L, Muntz HG, Melancon CH. Ovarian carcinoma diagnosis. Cancer. 2000;89:2068-2075.

4. Goff BA, Mandel LS, Dresden CW, et al. Development of an ovarian cancer symptom index: possibilities for earlier detection. Cancer. 2007;109:221-227.

5. Jelovac D, Armstrong DK. Recent progress in the diagnosis and treatment of ovarian cancer. CA Cancer J Clin. 2011;61:183-203.

6. van Nagell JR, Pavlik EJ. Ovarian cancer screening. Clin Obstet Gynecol. 2012;55:43-51.

7. Prorok PC. Evaluation of screening programs for the early detection of cancer. Natl Cancer Inst Stat Textbk Monogr. 1984;51:267-328.

8. Yancik R. Ovarian cancer age contrasts in incidence, histology, disease stage at diagnosis, and mortality. Cancer. 1993;71:517-523.

9. Quizk JT, Natarajan N, Mettlin CJ. Age-specific ovarian cancer incidence rate patterns in the United States. Gynecol Oncol. 2005;99:248-250.

10. Coch M, Gaedke H, Jenkins H. Family history of ovarian cancer patients: a case controlled study. Int J Epidemiol. 1989;18:782-785.

11. Lancaster JM, Powel CB, Knauff ND, et al. Statement in risk assessment for inherited gynecologic cancer predispositions. Gynecol Oncol. 2007;107:159-162.

12. Yang DH, Smith ER, Cohen C, et al. Molecular events associated with dysplastic morphologic transformations and initiation of ovarian tumorigenicity. Cancer. 2002;94:2380-2392.

13. Schildkraut JM, Bastos E, Berchuck A. Relationship between lifetime ovulating cycles and overexpression of mutant p53 in epithelial ovarian cancer. J Natl Cancer Inst. 1997;89:932-938.

14. Crum C, McKeon FD, Xian W. The oviduct and ovarian cancer: causality, clinical implications and targeted prevention. Clin Obstet Gynecol. 2012;55:24-35.

15. Carlson JW, Miron A, Jarboe EA, et al. Serous tubal intraepithelial carcinoma: its potential role in primary peritoneal serous carcinoma and serous cancer prevention. J Clin Oncol. 2008;26:4160-4165.

16. Kurman R, Shih I. The origin and pathogenesis of epithelial ovarian cancer: a proposed unifying theory. Am J Surg Pathol. 2010;34:433-443.

17. Salani R, Bristow RE. Surgical management of epithelial ovarian cancer. Clin Obstet Gynecol. 2013;55:75-95.

18. Pavlik EJ, DePriest PD, Gallion HH, et al. Ovarian volume related to age. Gynecol Oncol. 2000;77:410-422.

19. Ferrazzi E, Lissoni AA, Dordoni D, et al. Differentiation of small adnexal masses based on morphological characteristics of transvaginal sonographic imaging. J Ultrasound Med. 2005;24:1467-1473; quiz $1475-1476$.

20. Sassone AM, Timor-Tritsch IE, Artner A, Westhoff C, Warren WB. Transvaginal sonographic characterization of ovarian disease: evaluation of a new scoring system to predict ovarian malignancy. Obstet Gynecol. 1991;78:70-76. 
21. Ueland FR, DePriest PD, Pavlik EJ, Kryscio RJ, van Nagell JR Jr. Preoperative differentiation of malignant from benign ovarian tumors: the efficacy of morphologic indexing and Doppler flow sonography. Gynecol Oncol. 2003;91:46-50.

22. Modesitt SC, Pavlik EJ, Ueland FR, DePriest PD, Kryscio RJ, van Nagell JR Jr. Risk of malignancy in unilocular ovarian cystic tumors less than $10 \mathrm{~cm}$ in diameter. Obstet Gynecol. 2003;201: 594-599.

23. Saunders BS, Podzielinski I, Ware R, et al. Risk of malignancy in sonographically confirmed septated cystic ovarian tumors. Gynecol Oncol. 2010;118:278-282.

24. McDonald J, Doran S, DeSimone C, et al. Predicting risk of malignancy in adnexal masses. Obstet Gynecol. 2010;115:687-694.

25. Partridge E, Kreimer A, Grenlee RT, et al. Results from four rounds of ovarian cancer screening in a randomized trial. Obstet Gynecol. 2009;113:775-782.

26. Buys SS, Patridge E, Black A, et al. Effect of screening on ovarian cancer mortality: the Prostate, Lung, Colorectal and Ovarian (PLCO) Cancer Screening Randomized Controlled Trial. JAMA. 2011;305(22): 2295-2303.

27. Menon U, Gentry-Maharaj A, Hallett R, et al. Sensitivity and specificity of multimodal and ultrasound screening for ovarian cancer, and stage distribution of detected cancers: results of the prevalence screen of the UK Collaborative Trial of Ovarian Cancer Screening (UKCTOCS) Lancet Oncol. 2009;10:327-340.
28. Kobayashi H, Yamada Y, Sado T, et al. A randomized study of screening for ovarian cancer: a multicenter study in Japan. Int J Gynecol Cancer. 2008; 18:414-420.

29. van Nagell JR, Miller RW, DeSimone CP, et al. Long-term survival of women with epithelial ovarian cancer detected by ultrasonographic screening. Obstet Gynecol. 2011;118:1212-1221.

30. Pavlik EJ, van Nagell JR. Early detection of ovarian tumors using ultrasound. Womens Health (Lond Engl). 2013;9:39-57.

31. Cooper AL, Nelson DF, Doran S, et al. Long-term survival and cost of treatment in patients with stage IIIC epithelial ovarian cancer. Curr Womens Health Rev. 2009;5:44-50.

32. Elder J, Long R, Miller W, et al. Monitoring ovarian tumors using serial ultrasound with tumor morphology index. Gynecol Oncol. 2013;130:e94

33. Fleischer AC, Lyshchik A, Hirari M, Moore RD, Abramson RG, Fishman DA. Early detection of ovarian cancer with conventional and contrastenhanced transvaginal sonography: recent advances and potential improvements. J Oncol. 2012;2012:302858.

34. Fleischer A, Lyshchik A, Jones H, et al. Contrast-enhanced transvaginal sonography of benign versus malignant ovarian masses. $J$ Ultrasound Med. 2008;27:1011-1018; quiz 1019-1021.

35. Hirai M, Hirai Y, Tsuchida T, et al. Stage IA ovarian cancers comparison of sonographic findings and histopathologic types between patients with normal and elevated serum cancer antigen 125 levels J Ultrasound Med. 2011;30:943-952.
International Journal of Women's Health

\section{Publish your work in this journal}

The International Journal of Women's Health is an international, peerreviewed open-access journal publishing original research, reports, editorials, reviews and commentaries on all aspects of women's healthcare including gynecology, obstetrics, and breast cancer. The manuscript management system is completely online and includes

\section{Dovepress}

a very quick and fair peer-review system, which is all easy to use. Visit http://www.dovepress.com/testimonials.php to read real quotes from published authors. 